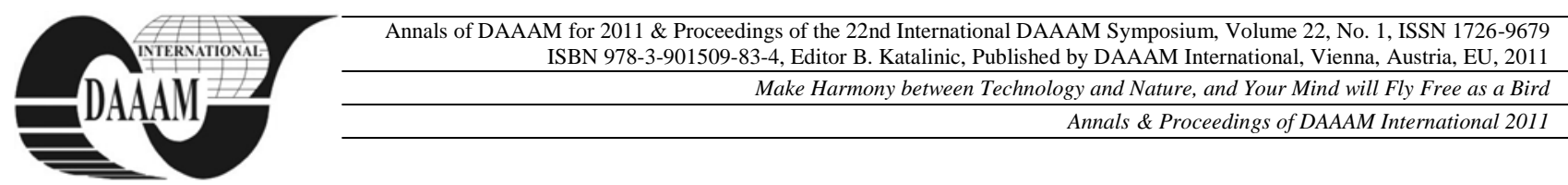

\title{
BUILDING AND TESTING OF A SIMPLE HIGH SPEED KINEMATICS MEASUREMENT SYSTEM
}

\author{
STANCIC, I[vo]; SUPUK, T[amara] \& ZANCHI, V[lasta]
}

\begin{abstract}
Ability of precise measurement and analysis of human movements is an essential tool for biomechanical research used in sports or clinical applications. Ideally, the measurement system should be non-invasive, safe to use, customizable and relatively inexpensive. In this paper design and evaluation of a high speed optical motion tracking and analysis system is described. It is designed to work with low cost components (LED markers, industrial cameras and PC). Static accuracy of the system is tested and results are presented in a form of error histogram. System is intended to be used in Biomechanical laboratories, where expensive commercial equipment is not available, but high precision $3 D$ kinematics measurement is mandatory.
\end{abstract}

Key words: kinematics measurement, active markers, biomechanics

\section{INTRODUCTION}

Human motion analysis becomes even more interesting because of its interdisciplinary nature and a wide range of applications. Last few decades of technological development created many types of kinematics measurement systems; magnetic, acoustical, mechanical, inertial and today mostly used optoelectronic devices (Winter, 2004; Medved, 2001). Modern motion capture and analysis systems were created for the needs of entertainment (game and film industry) and biomechanical research. Commercial optoelectronic systems like Optotrak or Vicon (Winter, 2004; Medved, 2001; Stancic 2009) that were constantly developing for last decades, show astonishing results; linear accuracy below millimetre and angular accuracy of only tenth of degree (Maletsky et al., 2007). Drawback of the mentioned systems is their high cost, and as a consequence they are not available to all biomechanical laboratories or studios. Hence, our idea was to present a procedure for building and testing a simple highspeed kinematics measurement system with abilities comparable to commercial systems.

Our system was based on a white visible-light LED marker, rather on an Infra Red (IR) marker, allowing simpler operation and troubleshooting in the measurement setup (Stancic, 2009). Another idea was to allow integration with the other laboratory equipment, using hardware triggers and easily accessible code developed in MATLAB (MATLAB, 2010). As described, main application of our system is marker tracking and calculation of a human body kinematics during movement. Kinematics analysis software was also developed. Software was able to automatically calculate, analyse and visualise all relevant kinematics data. An advanced image processing and analysis algorithm was used to achieve higher precision. Several subpixel algorithms, based on analysing of uneven distribution of a pixel light intensity around the marker centre, were developed, tested, and integrated inside our system.

Backbone of our system was a hi-speed industrial camera paired with the PC and a number of small, light-weight $3 \mathrm{~mm}$ markers (Fig. 1). Better performance should be achieved using IR markers and IR camera with a higher pixel accuracy (16 bit), but as this system is aimed for biomechanics laboratories, where simplicity and low measurement cost is more important than a sub-millimetre precisions or measurement speed in hundreds of $\mathrm{Hz}$, this system should be adequate.

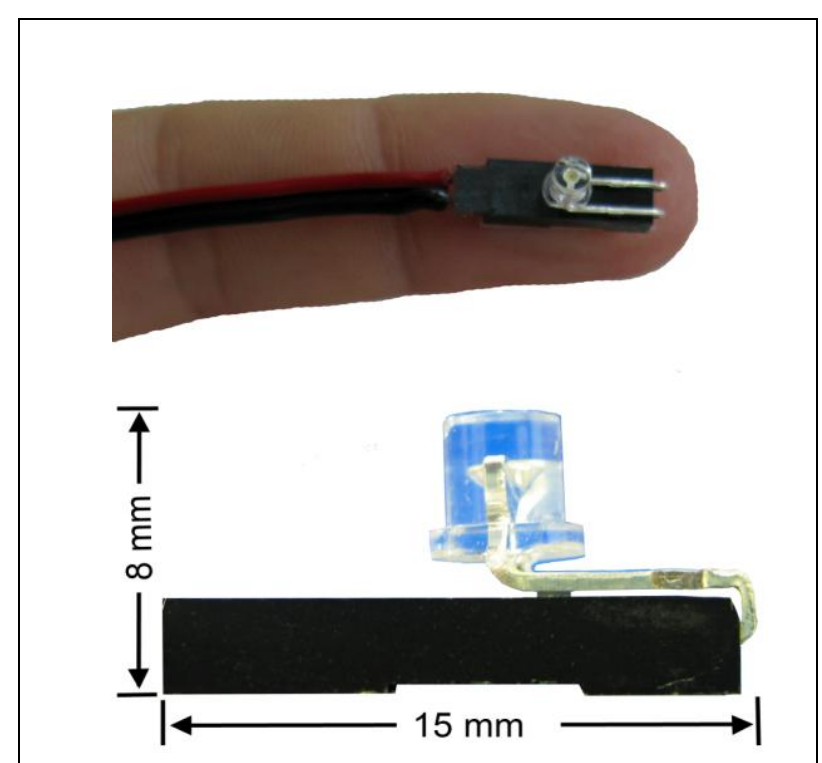

Fig. 1. Active Marker attached to fingertip (top), same marker with housing (bottom)

We have developed a method for testing the accuracy of markers in static conditions, where known position of a marker on rotating body was compared with the measured one (Lujan et al. 2005). Also, maximum density of markers in limited area which could be precisely tracked was benchmarked, showing that smaller body segments like fingers and foot could be successfully tracked.

\section{EXPERIMENTAL RESULTS}

Camera used for this work was a Basler $602 \mathrm{fc}$ fast industrial camera with Fujinon $12.5 \mathrm{~mm}$ lenses. Before actual measurement, we had to calibrate the system (cameras) to remove distortions and to obtain camera matrices (Shapiro \& Stockman, 2001; Trucco \& Verri, 1998). Physical camera calibration is commonly divided in to obtaining extrinsic and intrinsic parameters. Intrinsic parameters encompass focal length, image format, and principal point while extrinsic parameters are needed to transform object coordinates in to a camera coordinate frame, or in multi-camera system describe relationship between the cameras. Lenses distortion is decomposed in radial and decentring component, by knowing the distorted image coordinates corrected coordinates are recalculated. Camera model used by Heikkila et al allowed least square optimisation with distorted image coordinates, and in final achieved an accuracy of 1/50 of pixel size (Heikkila et al., 1997). 


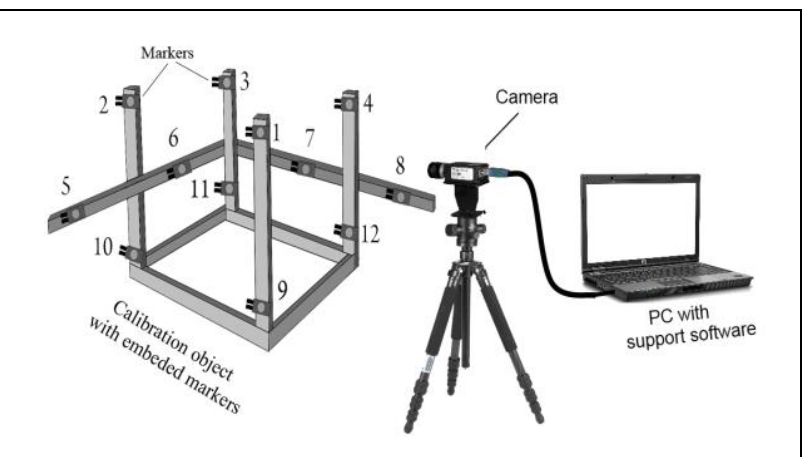

Fig. 2. Camera calibration setup

In next phase of calibration, after removing distortion, both intrinsic and extrinsic parameters are obtained for the whole multi-camera system. Calibration body was created using small metal table with extra two bars attached perpendicularly to the table legs, as shown on top right of the Figure 2. Calibration object takes $160 \times 90 \times 60 \mathrm{~cm}$ of space. Markers were arranged at the predefined spatial positions. All calibration procedures were preformed in a MATLAB as an integrated part of measurement software.

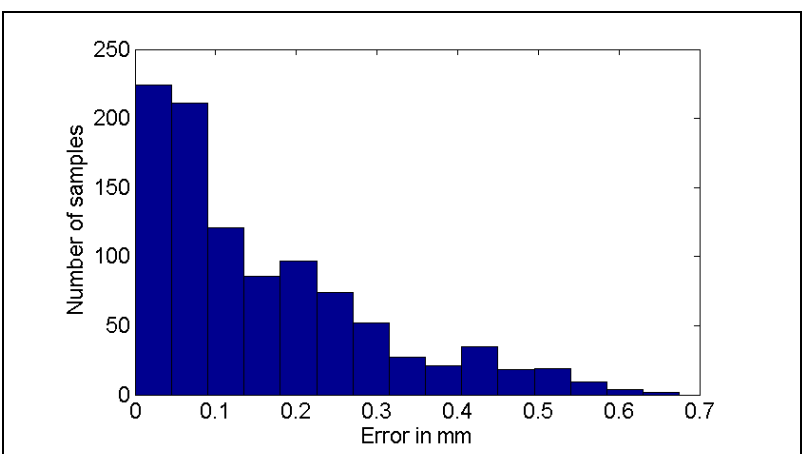

Fig. 3. Results of accuracy tests

After whole calibration procedure was finished, system was prepared for actual measurements. System was tested for static accuracy, with results presented in form of an error histogram shown in Figure 3. It is noticeable that most of the errors are inside $0.5 \mathrm{~mm}$ error range. Further statistical analysis reveals that more than $95 \%$ of results are inside $0.4912 \mathrm{~mm}$ error.

The next phase of measurement was trail on a living subject. Markers were placed on characteristic body landmarks of a subject's body. Subject was instructed to perform predefined movements (sit-to-stand motion, slow and fast walk etc.), as shown in Fig. 4. Result of measurement was video file or synchronized video files when a pair of cameras was used. Video files and systems calibration matrices were then used for marker detection and reconstruction software, and as an output table of coordinates for the each marker was created. Coordinates table was then used with a kinematics analysis software, and correct marker position was reconstructed for subjective and objective analysis of gait. Five markers were placed on subject's foot for tracking its kinematics during movements. Initial trails showed no problem caused by large number of fast markers inside small area. Only noticeable problem was marker occlusion, as software component for prediction of lost markers was not integrated. Highest possible speed achieved by the system was $300 \mathrm{~Hz}$, for $1 / 4$ of frame size, which allowed only tracking of few body segments (lower body).

The super-resolution technique was proposed to pre-process the image captured by cameras before performing the actual measurement algorithm. Picture produced by imaging sensor is a discrete data set given from continuous part of the object. The technique utilizes property of marker intensity distribution on a

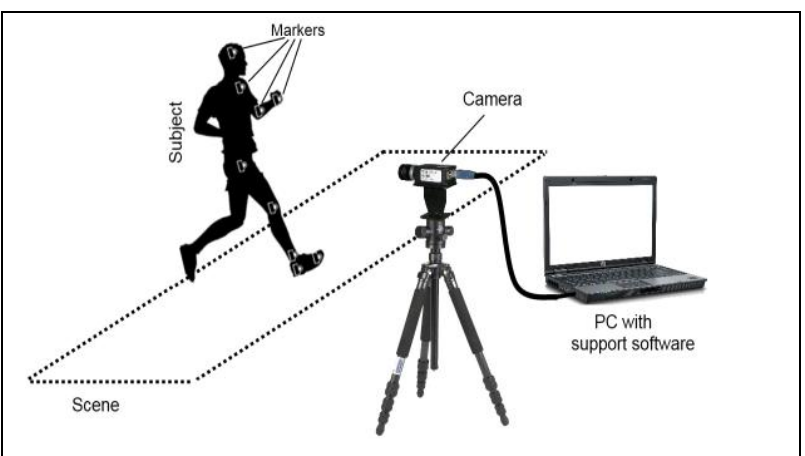

Fig. 4. Human kinematics measurement setup

captured image. In real conditions, when marker is not perfectly aligned with pixel centre, marker intensity distribution is not uniform on the edges. This property is used for creating Super resolution marker model, which was the core of our sub pixel accuracy resolution system.

\section{CONCLUSIONS}

The objective of this paper was to create and validate simple and cost effective kinematics measurement system with sub-pixel accuracy capabilities. LaBACS kinematics measurement system is a low cost system based on active visible light markers and fast industrial cameras. Algorithm incorporates components for camera lens distortion removal, system calibration, marker detection and tracking. High accuracy of marker 2D / 3D position reconstruction was achieved, and finally system is tested in working conditions for measuring human gait. Currently, the developed system is testing in Laboratory for Biomechanics Automation and Control System at University of Split

\section{ACKNOWLEDGMENTS}

This work is supported by Croatian Ministry of Science under the Project 023-0232006-1655 'Biomechanics of Human Motion, Control and Rehabilitation.

\section{REFERENCES}

Winter D. A., (2004), Biomechanics and Motor Control of Human Movement, Wiley, ISBN 978-0470398180, Canada

Medved V., (2001), Measurement of Human Locomotion, CRC Press, ISBN 978-0-8493-7675-7, UK

Stančić I., Borojević D., Šupuk T., (2009), Development and Testing of a Device for Human Kinematics Measurement. WSEAS transactions on systems, Vol 8, 1083-1092

Maletsky L. P., Sun J., Morton N. A., (2007), Accuracy Of An Optical Active-Marker System To Track The Relative Motion Of Rigid Bodies, Journal of Biomechanics, Vol. 40, 682-685

****MATLAB on line Users Guide. http://www.mathworks.com, Accessed on: 2011-09-20

Lujan T.J., Lake S.P., Plaizier T.A., Ellis B.J., Weiss J.A., (2005), Simultaneous measurement of three-dimensional joint kinematics and tissue strains with optical methods, Journal of Biomechanical Engineering, 193-197

Shapiro G., Stockman G. C., (2001), Computer vision, Prentice-Hall, ISBN 0-13-030796-3, USA

Trucco E., Verri A., (1998), Introductory Techniques for 3-D Computer Vision, ISBN 978-0132611084 Prentice Hall, USA

Heikkila J, Silven O., (1997), A Four-step Camera Calibration Procedure with Implicit Image Correction, IEEE Computer Society Conference on Computer Vision and Pattern Recognition (CVPR'97), pp 1106- 1112 